\title{
Voluntary approaches work in removal of artificial trans fats
}

In this letter by Barbara Gallani (BMJ 2011;343;d6115, doi:10. $1136 / \mathrm{bmj} . \mathrm{d} 6115)$ part of reference 1 was wrong . The reference was correct except for the elocator, which meant that it linked to the wrong article. The correct elocator is d5567, so the full reference should have read: "Coombes R. Trans fats: chasing a global ban. BMJ 2011;343:d5567, doi:10.1136/bmj.d5567. (7 September.)"

Cite this as: BMJ 2011;343:d6860

๑ BMJ Publishing Group Ltd 2011 